\title{
Mitigating the cognitive overload of contemporary argumentation-based collaboration settings
}

\author{
Nikos Karacapilidis \\ Computer Technology Institute \& Press «Diophantus» \\ and University of Patras, Greece \\ nikos@mech.upatras.gr
}

Nikos Karousos

Software Quality Research Group

Hellenic Open University, Patras, Greece

karousos@eap.gr

\author{
Manolis Tzagarakis \\ Computer Technology Institute \& Press «Diophantus» \\ and University of Patras, Greece \\ tzagara@upatras.gr \\ Spyros Christodoulou \\ Computer Technology Institute \& Press «Diophantus» \\ Patras, Greece \\ shristod@cti.gr
}

\begin{abstract}
We present CoPe_it!, an innovative web-based tool that complies with collaborative practices to provide members of diverse communities with the appropriate means to manage individual and collective knowledge during a complex sensemaking and/or decision-making session. We discuss the rationale behind the tool's development and present its features and functionalities that aim to efficiently deal with cognitive overload issues.
\end{abstract}

Keywords-web-based collaboration, visualization of collaboration, cognitive overload, collaborative decision making.

\section{INTRODUCTION}

Current advances in computing and Internet technologies, together with the advent of the Web 2.0 era, have resulted in the development of a plethora of online, publicly available environments such as blogs, discussion forums, wikis, and social networking applications. These offer people an unprecedented level of flexibility and convenience to participate in complex collaborative activities, such as long online debates of public interest about the greening of our planet through renewable energy sources or the design of a new product in a multinational company.

When engaged in argumentation-based collaborative environments, people have usually to go through some type of sorting, filtering, ranking and aggregation of the existing resources in order to facilitate sense-making and/or decision making. Yet, these activities are far from being easy. This is because collaboration settings are often associated with everincreasing amounts of multiple types of data, obtained from diverse sources that often have a low signal-to-noise ratio for addressing the problem at hand. In turn, these data may vary in terms of subjectivity, can be of diverse level as far as human understanding and machine interpretation are concerned and are interconnected in vague or explicit ways. Data and their interconnections often reveal social networks and social interactions of different patterns. In such situations, collaboration becomes cognitively complex.

This publication has been produced in the context of the EU Collaborative Project "DICODE - Mastering Data-Intensive Collaboration and Decision Making" which is co-funded by the European Commission under the contract FP7-ICT-257184. This publication reflects only the author's views and the Community is not liable for any use that may be made of the information contained therein.
Cognitive complexity may be defined as the sum of the factors that makes things hard to see, use, grasp, and understand [1]. It may be considered as a function of the content, structure and amount of knowledge needed to perform a specific task [2]. Admittedly, increase in complexity places heavy demands on working memory and leads to an increase in cognitive strain, which often leads to lower performance [3].

Generally speaking, cognitive overload is an endemic problem to collaborative activities. Major reasons contributing to this problem include the growing number of information items, the uncertainty and diversity of information, the increasing number of alternatives, the intensity and ambiguity of information, the information quality, the overabundance of irrelevant information and the lack of appropriate search mechanisms $[4,5]$. The above bring up the need for innovative software tools that - by design - adopt countermeasures against information overload and cognitive complexity and which have already been reported in the literature [6-8].

Existing collaboration support systems mainly focus on the expression and visualization of arguments. However, their features and functionalities are limited, they pay no or limited attention to data and knowledge management issues, they are mostly tested in academic environments, they are not interconnected with other tools, and they do not efficiently tackle the technological and social dimensions of cognitivelycomplex collaboration. When compared against available cognitive overload countermeasures [4], the vast majority of these tools do not offer services to aggregate, categorize and structure information, have static and limited capabilities to support visualization, do not provide various levels of detail and lack mechanisms to support awareness and information filtering that could help proactively in processing the available information. To sum up, existing approaches neglect to address cognitive overload concerns. In this context, our work focuses on the development of a web-based tool, namely CoPe_it! (http://copeit.cti.gr), which is capable to tackle the diversity and complexity of the above issues, the ultimate goals being to 
make it easier for users to follow the evolution of an ongoing collaboration, comprehend it in its entirety, and meaningfully aggregate data in order to resolve the issue under consideration.

\section{REQUIREMENTS AND CHALLENGES}

Towards meeting the challenges involved in cognitive complex argumentation based collaboration, we have performed a series of interviews with members of diverse communities in order to identify the major issues they face during their ordinary practices. With respect to cognitive complexity, major issues identified were:

\section{A. Information overload}

This is primarily due to the extensive and uncontrolled exchange of diverse types of data and knowledge resources. For instance, such a situation may appear during the exchange of numerous ideas about the solution of a public issue, which is accompanied by the exchange of big volumes of positions and arguments in favor or against each solution.

\section{B. Difficulty in monitoring social behavior}

The representation and visualization of social structures, relationships and interactions taking place in a collaborative environment with multiple stakeholders are also of major importance. This is associated to the perception and modeling of actors, groups and organizations in the diversity of collaborative contexts. A problem to be addressed is to provide the means to appropriately represent and manage user and group profiles, as well as social relationships given that they are not static but changing over time.

\section{Diversity of collaboration modes}

Interviews indicated that the evolution of a collaboration session proceeds incrementally; ideas, comments, or any other type of collaboration objects are exchanged and elaborated, and new knowledge emerges slowly. When members of a community participate in a collaborative session, enforced formality may require them to specify their knowledge before it is fully formed. Such emergence cannot be attained when the collaborative environment enforces a formal model from the beginning. On the other hand, formalization is required in order to ensure the environment's capability to support decision making or estimate the present state of the collaboration

\section{Expression of tacit knowledge}

A community of people is actually an environment where tacit knowledge (i.e. knowledge that the members do not know they possess or knowledge that members cannot express with the means provided) predominantly exists and dynamically evolves.

\section{E. Difficulty in exploiting and integrating legacy resources}

Many resources required during a collaborative session have either been used in previous sessions or reside outside the members' working environment (e.g. in e-mailing lists or web forums). Moreover, outcomes of past collaboration activities should be able to be reused as input in subsequent collaborative sessions. Yet, such functionality must be provided in ways that do not disrupt or impede an ongoing collaboration.

\section{F. Data processing and decision making support}

In the settings under consideration, timely processing of data related to both the social context and social behavior is required. Such processing will significantly aid the members of a community to conclude the issue at hand, extract meaningful knowledge and reach a decision. This means that their environment needs to interpret the knowledge item types and their interrelationships in order to proactively suggest trends or even aggregate data and calculate the outcome of a collaborative session.

The above issues delineated some categories of crucial requirements to be met during the development of CoPe_it!.

\section{THE PROPOSED SOLUTION}

CoPe_it! allows for distributed, synchronous or asynchronous, collaboration over the Web. Our overall approach is the result of action research studies [9] concerning the improvement of practices, strategies and knowledge in diverse cognitively-complex collaborative environments. The research method adopted for the development of CoPe_it! follows the design science paradigm [10]. To appropriately tackle the issues identified in the previous section, CoPe_it! builds on an integrated consideration and exploitation of the concepts listed below.

\section{A. Incremental formalization}

In existing technologies and systems supporting argumentation-based collaboration, the interaction of users is regulated by procedures that prescribe and - at the same time constrain their work. However, there is much evidence that sophisticated approaches and techniques often resulted in failures $[11,12]$. This is often due to the extra time and effort that users need to spend in order to get acquainted with the system, the associated disruption of the users' usual workflow [13], as well as to the 'error prone and difficult to correct when done wrong' character of formal approaches [14].

Furthermore, formal approaches impose a structure which in complex contexts is not mature enough to accommodate the management of huge amounts of data coming from diverse sources. They do not allow users to elaborate and digest these data at their own pace, according to the evolution of the collaboration. Lack of flexible, situation-oriented processing of information may lead to cognitive overload. Thus, a varying level of formality should be considered.

The above advocate an incremental formalization approach, which has been adopted in the development of CoPe_it!. In our approach, formality and the level of knowledge structuring is not considered as a predefined and rigid property, but rather as an adaptable aspect that can be modified to meet the needs of the tasks at hand. By the term formality, we refer to the rules enforced by the system, with which all user actions must comply. Allowing formality to vary within the collaboration space, incremental formalization, i.e. a stepwise and controlled evolution from a mere collection of individual ideas and 
resources to the production of highly contextualized and interrelated knowledge artifacts, can be achieved. This enables the adaptation of a collaboration support system to meet the cognitive overload issues that occur each time. This evolution is associated with a set of functionalities that are ordered in terms of formality level.

\section{B. Visualization and reasoning}

It has been widely argued that visualization of argumentation conducted by a group of experts working collaboratively towards solving a problem can facilitate the overall process in many ways, such as in explicating and sharing individual representations of the problem, in maintaining focus on the overall process, as well as in maintaining consistency and in increasing plausibility and accuracy [15]. Moreover, it leads to the enhancement of the group's collective knowledge. For the above reasons, visualization issues received much attention while shaping the proposed solution.

In CoPe_it!, different visualizations of the collaboration are provided; these are called "projections" and provide the means to achieve the desired incremental formalization of collaboration. A projection can be defined as a particular representation of the collaboration space, in which a consistent set of abstractions able to solve a particular organizational problem during argumentation-based collaboration is available. With the term abstraction, we refer to the particular data and knowledge items, relationships and actions that are supported through a particular projection, and with which a particular problem can be represented, elaborated and be solved.

In CoPe_it!, a particular collaboration space can be viewed in different projections that end-users individually may specify. CoPe_it! enables switching from a projection to another, during which abstractions of a certain formality level are transformed to the appropriate abstractions of another formality level. This transformation is rule-based; such rules can be defined by users and/or the facilitator of the collaboration and reflect the evolution of a community's collaboration needs.

Each projection of the collaboration space provides the necessary mechanisms to support a particular level of formality. The more informal a projection is, the more easiness-of-use is implied; at the same time, the actions that users may perform are intuitive and not time consuming. Informality is associated with generic types of actions and resources, as well as implicit relationships between them that users may create expressing agreement, disagreement, support, request for refinement, contradiction etc. The aim of an informal projection of the collaboration space is to provide users the means to structure and organize data and knowledge items easily, and in a way that conveys semantics to them. Informal projections exhibit a low level of formality.

On the other, decision making processes can be better supported in environments that exhibit a high level of formality. The more formal projections of a collaboration space come to serve such needs. More formal projections are associated with less easiness-of-use; actions permitted are less intuitive and more time consuming. Formality is associated with fixed types of actions, as well as explicit relationships between them.

\section{Information triage}

Our solution builds extensively on the information triage process [16], i.e. the process of sorting and organizing through numerous relevant materials and organizing them to meet the task at hand. During such a process, users must effortlessly scan, locate, browse, update and structure knowledge resources that may be incomplete, while the resulting structures may be subject to rapid and numerous changes. Information triage related functionalities enable users to meaningfully organize the big volumes of data and knowledge items in a collaborative setting.

The informal projection of a collaborative workspace in CoPe it! is fully in line with the above. Drawing upon successful technologies coming from the area of spatial hypertext [16], the informal projection of CoPe_it! adopts a spatial metaphor to depict collaboration in a 2.5 -dimensional space (the space is considered 2.5-dimensional, and not 2dimensional, because it permits overlap of the items; the tool is aware of which items overlap, as well as of various spatial proximity issues). Users are incrementally processing information and are not forced to predefined structural commitments. The related features and functionalities of CoPe_it! enable users to create and organize information by making use of spatial relationships and structures, giving them the freedom to express relationships among information items through spatial proximity and visual cues. Such cues are related to the linking of collaboration items (e.g. coloring and thickness of the respective links) and the drawing of colored rectangles to cluster related items.

As highlighted above, the informal projection of a collaborative workspace in $\mathrm{CoPe}$ it! permits an ordinary and unconditioned evolution of data and knowledge structures. This projection also provides abstraction mechanisms that allow the creation of new abstractions out of existing ones that include annotation, aggregation and specialization. Such mechanisms constitute valuable means to mitigate cognitive overload by enabling the structuring of the collaboration space. The tool enables information triage related activities to be conducted either collaboratively or individually.

\section{Filtering, overviews, history and awareness}

CoPe_it! provides a number of functionalities to mitigate information overload and the associated cognitive complexity. These include filtering mechanisms, which permit users to display on the collaboration space only those items that fulfill specific criteria. The tool's workspace also features a 'minimap' providing an overview of its contents, thus enabling easy and quick navigation. A 'history mechanism' allows users to follow the evolution of a workspace and enables them to see how the workspace changed in time (such as what items have been uploaded, which relationships have been created etc.). In addition, awareness mechanisms allow participants to get informed about the actions of other collaborators. 


\section{E. Exploitation of legacy resources}

$\mathrm{CoPe}$ it! reduces the overhead of entering information by allowing the reuse of existing resources. Generally speaking, when legacy resources have to be reused during a collaborative session, complexity is increased. This is not only due to the additional amount of data involved, but also to the conceptual overhead and distractions imposed to the user from switching among applications and environments. One way of dealing with this situation is to enable the ubiquitous access of legacy resources from within the collaboration environment by seamlessly integrating the systems involved. Towards this direction, we have achieved interoperability between CoPe_it! and a number of applications that include web-based forums, search engines and existing argumentation-based collaboration tools (e.g. Compendium).

\section{F. Social networking}

Management of social structures, interactions and relationships is also critical in a complex e-collaboration framework. Applications and projects dealing with social relationships mainly support explicit and abstract structures. However, social structures may gain from the expertise of structure domain research, including various structure abstractions or ways for implicit structuring. Another issue to be addressed concerns the elaboration of social relationships in their contexts, that is, how they relate to assets, locations, or change over time. Social network analysis [17] has to be extensively used to find who is depending on whom in a network. Such an analysis will also help to detect hidden hierarchy of social networks.

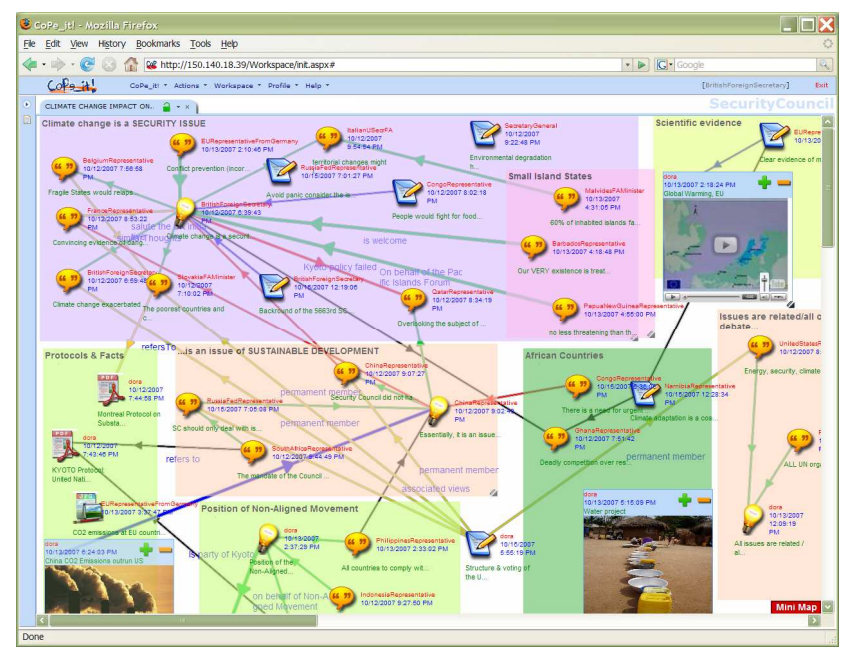

Fig. 1. An instance of the CoPe_it! environment (details about the specific use case can be found in [18]).

\section{CONCLUDING REMARKS}

CoPe_it! has been already introduced in diverse collaboration settings for a series of pilot applications (an instance of the CoPe_it! environment is shown in Fig. 1). The results of the first evaluation phase were very encouraging (details about these results appear in [18]). Summarizing, we argue that the proposed approach covers fully the user requirements analyzed in this article. CoPe_it! attempts to address the evolution of argumentation-based collaboration and facilitate the associated sense-making and decision making processes - by providing a number of projections, each one supporting a particular level of formality, ranging from informal (more human and less interpretable) to formal (more machine and less human interpretable) ones. Due to its inherent scalability, it is able to fully support the evolution of a cognitively-complex collaboration session, while it provides the means for addressing the issues related to the formality needed in collaborative knowledge building systems.

\section{REFERENCES}

[1] D. Batra, "Cognitive complexity in data modeling: causes and recommendations", Requir. Eng., vol. 12, 2007, pp.231-244.

[2] D. Kieras and P. Polson, "An approach to the formal analysis of user complexity”, Int J Man Mach Stud 22, 1985, pp. 365-394.

[3] J. S. B. T. Evans, "Thinking and reasoning: psychological approaches", Routledge \& Kegan Paul, Boston, 1983.

[4] M. Eppler and J. Mengis, "The Concept of Information Overload: A Review of Literature from Organization Science, Accounting, Marketing, MIS, and Related Disciplines", The Information Society, vol. 20, no 5, 2004, pp. 325-344.

[5] S. C. Schneider, "Information overload: Causes and consequences. Human Systems Management 7”, 1987, pp. 143-153.

[6] D. L. Scammon, "Information load and consumers", Journal of Consumer Research 4, 1977, pp. 148-156.

[7] M. Grise, and R.B. Gallupe, "Information overload: Addressing the productivity paradox in face-to-face electronic meetings", Journal of Management Information Systems 16(3), 1999/2000, pp.157-185.

[8] S. Y. Chan, "The use of graphs as decision aids in relation to information overload and managerial decision quality", Journal of Information Science 6, 2001, pp: 417-426.

[9] P. Checkland and S. Holwell, "Action Research: Its Nature and Validity", Systemic Practice and Action Research, vol. 11, no 1, 1998, pp. 9-21.

[10] A.R. Hevner, S.T. March, J. Park and S. Ram, "Design Science in Information Systems Research”, MIS Quarterly, vol. 28, no 1, 2004, pp. $75-105$.

[11] F.M. Shipman and C.C. Marshall, "Formality Considered Harmful: Issues, Experiences, Emerging Themes, and Directions", Tech. Rep. ISTL-CSA-94-08-02, Xerox Palo Alto Research Center, 1994.

[12] F.M. Shipman and R. McCall, "Supporting knowledge-base evolution with incremental formalization", Proc. CHI '94 Conference, 1994, pp. 285-291.

[13] G. Fischer, A.C. Lemke, R. McCall and A. Morch, "Making Argumentation Serve Design", Human Computer Interaction, vol. 6, no 3-4, 1991, pp. 393-419.

[14] F. Halasz, "Reflections on NoteCards: Seven Issues for the Next Generation of Hypermedia Systems", Communications of the ACM, vol. 31, no 7, 1988, pp. 836-852.

[15] P. Kirschner, S. Buckingham Shum and C. Carr, "Visualizing Argumentation: Software Tools for Collaborative and Educational Sense-Making”, Springer, 2003.

[16] C. Marshall and F.M. Shipman, "Spatial Hypertext and the Practice of Information Triage", Proc. 8th ACM Conference on Hypertext, 1997, pp. 124-133.

[17] M. Castells, "The Network Society: A Cross-cultural Perspective", Edward Elgar, 2004

[18] N. Karacapilidis, M. Tzagarakis, N. Karousos, G. Gkotsis, V. Kallistros, S. Christodoulou, C. Mettouris and D. Nousia, "Tackling cognitivelycomplex collaboration with CoPe_it",. International Journal of WebBased Learning and Teaching Technologies, vol. 4, no 3, 2009, pp. 22 38. 